\title{
Neighbor Discovery in a Wireless Sensor Network: Multipacket Reception Capability and Physical-Layer Signal Processing
}

\author{
Jeongho Jeon and Anthony Ephremides
}

\begin{abstract}
In randomly deployed networks, such as sensor networks, an important problem for each node is to discover its neighbor nodes so that the connectivity amongst nodes can be established. In this paper, we consider this problem by incorporating the physical layer parameters in contrast to the most of the previous work which assumed a collision channel. Specifically, the pilot signals that nodes transmit are successfully decoded if the strength of the received signal relative to the interference is sufficiently high. Thus, each node must extract signal parameter information from the superposition of an unknown number of received signals. This problem falls naturally in the purview of random set theory (RST) which generalizes standard probability theory by assigning sets, rather than values, to random outcomes. The contributions in the paper are twofold: first, we introduce the realistic effect of physical layer considerations in the evaluation of the performance of logical discovery algorithms; such an introduction is necessary for the accurate assessment of how an algorithm performs. Secondly, given the double uncertainty of the environment (that is, the lack of knowledge of the number of neighbors along with the lack of knowledge of the individual signal parameters), we adopt the viewpoint of RST and demonstrate its advantage relative to classical matched filter detection method.
\end{abstract}

Index Terms-wireless sensor network, neighbor discovery, multipacket reception, random set theory

\section{INTRODUCTION}

$\mathbf{W}$ IRELESS sensor networks are gaining great attention due to its versatility in civilian/military applications such as environmental monitoring and target detection/tracking in a cost-effective manner. In such applications, a large number of sensors are randomly deployed over the region of interest and, presumably, neighbor discovery is the first and foremost process to run after the deployment to form a network whose connectivity greatly affects the performance of subsequent network operations over the entire life span. The challenge is compounded by the fact that neighbor discovery has to be done without any a priori knowledge on the random deployment or any communication infrastructures.

Neighbor discovery in wireless networks is defined to be the process to identify a set of nodes with which a node can communicate, and it has been addressed by several authors [1]-[4]. In [1], a simple ALOHA-like neighbor discovery algorithm

The material in this paper was presented in part at the 48th Annual Allerton Conference on Communication, Control, and Computing (Monticello, IL), Sept. 2010.

The authors are with the Department of Electrical and Computer Engineering and the Institute for Systems Research, University of Maryland, College Park, MD 20742 USA (e-mail: jeongho@umd.edu; etony@umd.edu). was proposed in which each node randomly transmit/listen in each time slot and analyzed for both synchronous and asynchronous timing cases. This type of discovery algorithm based on the random access protocol is well suited for randomly distributed wireless networks. In [2], similar neighbor discovery algorithm was considered and the expected time to find neighbors was obtained. In [3], a gossip-based algorithm was proposed in which each node transmits a table of gossip data (which is the list of neighbors that it has discovered so far and their locations) in a random direction using directional antennas. In [4], a family of probabilistic protocols, called birthday protocols, have been proposed to initiate the randomly deployed wireless networks. From a physical-layer point of view, however, the previous works are extremely limited due to the use of collision channel model. Under this model, if more than one nodes transmit at the same time, none of them are successful. However, it is too pessimistic in the sense that a transmission may succeed even in the presence of interference which is called capture effect [5]-[9]. We, thus, claim that the performance of neighbor discovery algorithm has been quite underestimated so far due to the use of unrealistic channel model, and correct reassessment of the discovery algorithm is required.

In this paper, we consider a shared channel and nodes with multipacket reception capability in which a transmission is successful if the received signal-to-interference-plus-noiseratio (SINR) exceeds a certain threshold. Specifically, under the discovery algorithm proposed in [1], we obtain the expression for the expected number of successful receptions per slot at a given SINR threshold and find the optimal transmission probability which maximizes the expected number of successful receptions. We note, however, that for a given modulation scheme and target bit error rate (BER), the data rate is an increasing function of the SINR threshold. Therefore, for a fair comparison, we normalize the slot duration as the unit time and obtain the expected number of successful receptions per second. After that, the performance of the discovery algorithm over multiple slots is studied which is useful, for example, in deciding the duration of the discovery process.

In dealing with multipacket reception capability, the physical layer signal processing issues cannot be overlooked. This is because the received signal at each time slot is the superposition of the signals transmitted from random set of nodes and noise. Therefore, determining the existence of a signal from a particular node itself is not an easy task. For this problem, we first present the classical matched filter method 
which fundamentally treats the interference as noise. As an alternative, a more accurate method can be envisioned in an additional cost of complexity. Since the number of transmitters and their entities are all unknown, we adopt the viewpoint of random set theory (RST: see Appendix A and references therein) and propose RST-based method for detecting the transmitting nodes in each time slot [10]-[16]. Besides, it is also possible with RST to estimate additional parameters of transmitted signals such as signal amplitudes and phase.

This paper is organized as follows. The next section describes the basic assumptions, establishes the notation, and presents the signal model. In Section III details on the neighbor discovery algorithm is presented and the early termination of the discovery process is discussed. In Section IV we analyze the chosen discovery algorithm under the SINRbased model. In Section $\mathrm{V}$, physical layer signal processing solutions are delivered. Specifically, we obtain matched filter detector for neighbor discovery and, as an alternative, RSTbased maximum a posteriori (MAP) estimator is proposed. In Section VI numerical results are presented. Finally, we draw conclusions in Section VII

\section{System MODEL}

We consider a time-slotted wireless sensor network which is deployed over a region of interest such as large tactical area for target detection or vast rural area for environmental observation. In such scenarios, a large number of sensor nodes are released from an airplane. For a large number of nodes over a large area, the locations of nodes are modeled by a homogeneous, two-dimensional Poisson point process with intensity $\lambda$ which is the average number of nodes per unit area. Therefore, the number of nodes in a unit area follows Poisson distribution with parameter $\lambda$. As is well known, a spatial Poisson process on the plane, conditioned on a given number of nodes within a given area, yields the uniform distribution of these nodes in that area. As a result, for example, if a node's discovery region is modeled as a circle of radius $R_{0}$, the cumulative probability distribution on the distance from the node to the other nodes in the region is given by

$$
F_{r}(x)=\left\{\begin{array}{cl}
0, & \text { if } x<0 \\
\left(\frac{x}{R_{0}}\right)^{2}, & \text { if } 0 \leq x \leq R_{0} \\
1, & \text { if } x>R_{0}
\end{array}\right.
$$

The signal received by a node at time slot $t$ (if the node is listening) is given by

$$
\mathbf{y}_{t}=\sum_{k \in \mathbf{I}_{t}} g_{t}^{k} \mathbf{s}_{t}^{k}+\mathbf{n}_{t}
$$

where $\mathbf{I}_{t}$ is the set of transmitting neighbors, $g_{t}^{k}$ is the complex amplitude of the signal received from the $k$-th node, $\mathbf{s}_{t}^{k}$ is the signal transmitted from the $k$-th node which is the message multiplied by the signature sequence bitwise, and $\mathbf{n}_{t}$ is a random noise. We assume that the signatures of all nodes are known to each other by assuming that they share an identical key generator which can be implemented using a linear feedback shift register. If the length of signature is $L$ and each node transmits a 1-bit known message, then $\mathbf{y}_{t}=\left\{y_{t, 1}, \ldots, y_{t, L}\right\}^{T}$, $\mathbf{s}_{t}^{k}=\left\{s_{t, 1}^{k}, \ldots, s_{t, L}^{k}\right\}^{T}$ which is equal to the signature sequence, and $\mathbf{n}_{t}=\left\{n_{t, 1}, \ldots, n_{t, L}\right\}^{T}$, where the symbol $T$ denotes vector transpose. The noise samples in $\mathbf{n}_{t}$ are assumed to be independent and identically distributed (i.i.d.) with the normal distribution $\mathcal{N}(0, N)$ where $N$ is the noise power which is the product of noise spectral density $N_{0}$ and bandwidth $B$. The complex amplitude $g_{t}^{k}$ is of the form $g_{t}^{k}=\sqrt{G\left(1+r_{k}\right)^{-\eta}} \psi_{t}^{k}$, where $G$ is the transmission power, $r_{k}$ is the distance from the $k$-th node to the receiver, $\eta$ is the path loss exponent, and $\psi_{t}^{k}$ is the channel fading coefficient which is modeled by i.i.d. standard circular symmetric Gaussian random process [17].

Denote by $\mathcal{J}$ and $J$ the set of neighbors and the number of neighbors of a node, respectively, and we suppressed the particular node index for notational brevity. The SINR of node $k$ at time slot $t$ (if the $k$-th node is transmitting) is given by

$$
\operatorname{SINR}_{t}^{k}=\frac{P_{r x, k}}{\sum_{i \in \mathbf{I}_{t}, i \neq k} P_{r x, i}+N}
$$

where $P_{r x, k}$ is the received signal power from the $k$-th transmitter. Note that the distances $r_{k}(k=1, \ldots, J)$ are i.i.d. under the Poisson point process modeling. Also the channel fading coefficients $\psi_{t}^{k}$ are i.i.d., and by further assuming that $r_{k}$ and $\psi_{t}^{k}$ are mutually independent, the received signal powers $P_{r x, k}(k=1, \ldots, J)$ are i.i.d. as well, and the common cumulative distribution function of the received signal power is obtained by [6]

$$
F_{P}(x)=1-\int_{0}^{\infty} F_{r}\left(\left(\frac{\omega G}{x}\right)^{\frac{1}{\eta}}-1\right) f_{|\psi|^{2}}(\omega) d \omega
$$

where $f_{|\psi|^{2}}(\cdot)$ is the probability density of the squared magnitude of the stationary fading process which is an exponential with unit mean. The transmission of the $k$-th node is said to be successful if

$$
\operatorname{SINR}_{t}^{k} \geq \tau
$$

where the threshold $\tau$ depends on parameters such as data rate and target BER [18]. Note that, if $\tau<1$, it is possible for two or more signals to simultaneously satisfy (4), and if $\tau \geq 1$, at most one signal having the highest SINR may satisfy (4). If $\tau$ goes to $\infty$ and we neglect the noise effect, the criterion in (4) is equivalent to the collision channel model, and if $\tau$ goes to 0 , all transmissions will be successful, but at the same time, the data rate goes to 0 .

\section{The Neighbor Discovery Algorithm}

\section{A. Description of the Neighbor Discovery Algorithm}

An ALOHA-like neighbor discovery algorithm proposed in [1] is reconsidered in which each node transmits with probability $p_{\mathrm{T}}$ or listens with probability $1-p_{\mathrm{T}}$. The transmission probability $p_{\mathrm{T}}$ and the transmission power $G$ are identical for all nodes; all these simplifying assumptions were made to reduce non-essential complexities. Notice that a plain method such as the periodic beaconing can be thought but such a static approach would certainly fail in a randomly deployed network which has irregular node density. Under the chosen discovery 
algorithm, in order for a node to be discovered by some other node, the former should transmit and the other should listen at the same time. On top of that, the transmission must be successful which requires a certain criterion; in the collision channel, the criterion is that there is only one transmission at that time slot. However, we adopt a more realistic SINR criterion for success and optimize the transmission probability which has been quite underestimated so far due to the use of collision channel model.

\section{B. Considerations on Early Termination}

Obviously, the marginal revenue of running the discovery algorithm diminishes as time goes and, because sensors are usually assumed to be battery-powered, it is needed to consider an early termination of the discovery process to prolong the lifetime of the network. Several metrics can be envisioned as a criterion for the early termination. A simple criterion is to terminate when a node has discovered a predetermined number of neighbors. In the case of nodes located at the sparsely populated areas, however, this may not be satisfied until the end of the discovery process (even if all the neighbors are discovered early). Alternatively, one may want to allow an early termination if a node has discovered a predetermined fraction of neighbors. However, because each node does not know a priori how many neighbors it has, this criterion is untestable. As will be seen in the next section, as time goes, the set of discovered neighbors in each time slot will overlap with the previously discovered ones. Therefore, if a node do not find any new neighbors for a sufficiently large number of slots, then it can be regarded that all the neighbors have been discovered. Hence, an early termination can be declared if a node does not receive any new messages during a predetermined number of time slots.

\section{Analysis of the Discovery Algorithm With the MULTIPACKET RECEPTION CAPABILITY}

\section{A. Optimal Transmission Probability}

We start by deriving the expected number of successful receptions in each time slot as a function of SINR threshold $\tau$. After that the transmission probability $p_{\mathrm{T}}$ is set to maximize it. We first denote by $\mathbf{I}_{t}^{s}\left(\subseteq \mathbf{I}_{t}\right)$ the set of transmitting neighbors satisfying the SINR criterion in (4). Then, the expected number of successful receptions by one node is expressed as 1

$$
\begin{aligned}
E\left[\left|\mathbf{I}_{t}^{s}\right|\right] & =\operatorname{Pr}\{\text { a node is listening }\} \sum_{n=1}^{J} \operatorname{Pr}\left\{\left|\mathbf{I}_{t}\right|=n\right\} S_{n} \\
& =\sum_{n=1}^{J}\left(\begin{array}{l}
J \\
n
\end{array}\right) p_{\mathrm{T}}{ }^{n}\left(1-p_{\mathrm{T}}\right)^{J-n+1} S_{n}
\end{aligned}
$$

where $S_{n}$ is the expected number of successful receptions given $n(\geq 1)$ simultaneous transmissions and is obtained by

$$
S_{n}=n \operatorname{Pr}\left\{\operatorname{SINR}_{t}^{1}>\tau|| \mathbf{I}_{t} \mid=n, 1 \in \mathbf{I}_{t}\right\}
$$

where $\operatorname{SINR}_{t}^{1}$ is the SINR of the first transmitter [6]. Note that (6) follows from the assumption that the received signal

\footnotetext{
${ }^{1}|\cdot|$ is the cardinality of a set.
}

powers $P_{r x, k}(k=1, \ldots, J)$ are i.i.d. and, thus, the first transmitter needs not be the closest one to the receiver. It is computed as

$$
\begin{aligned}
& \operatorname{Pr}\left\{\operatorname{SINR}_{t}^{1}>\tau|| \mathbf{I}_{t} \mid=n, 1 \in \mathbf{I}_{t}\right\} \\
& =1-\int_{0}^{\infty} \cdots \int_{0}^{\infty} F_{P}\left(\tau \sum_{i=2}^{n} x_{i}\right) d F_{P}\left(x_{2}\right) \cdots d F_{P}\left(x_{n}\right)
\end{aligned}
$$

where the noise effect was neglected for simplicity. Consequently, in principle, we can find the optimal transmission probability that maximizes the expected number of successful receptions.

Example 4.1: As an illustration of the use of above equations, consider a simple example in which there are three nodes and they are within the radio range of each other. Since all three nodes have two neighbors, the expected number of successful receptions by one node is given by

$$
\begin{gathered}
E\left[\left|\mathbf{I}_{t}^{s}\right|\right]=2 p_{\mathrm{T}}\left(1-p_{\mathrm{T}}\right)^{2} \operatorname{Pr}\left\{\operatorname{SINR}_{t}^{1}>\tau|| \mathbf{I}_{t} \mid=1,1 \in \mathbf{I}_{t}\right\} \\
+2 p_{\mathrm{T}}{ }^{2}\left(1-p_{\mathrm{T}}\right) \operatorname{Pr}\left\{\operatorname{SINR}_{t}^{1}>\tau|| \mathbf{I}_{t} \mid=2,1 \in \mathbf{I}_{t}\right\}
\end{gathered}
$$

Let us further consider a simplified path loss model in which the received signal power is given by $P_{r x, k}=r_{k}^{-\eta}$. Then, it can be easily shown that for $\tau<1$,

$$
E\left[\left|\mathbf{I}_{t}^{s}\right|\right]=\tau^{\frac{2}{\eta}} p_{\mathrm{T}}^{3}-\left(2+\tau^{\frac{2}{\eta}}\right) p_{\mathrm{T}}^{2}+2 p_{\mathrm{T}}
$$

and for $\tau \geq 1$, ff

$$
E\left[\left|\mathbf{I}_{t}^{s}\right|\right]=\left(2-\tau^{-\frac{2}{\eta}}\right) p_{\mathrm{T}}{ }^{3}+\left(\tau^{-\frac{2}{\eta}}-4\right) p_{\mathrm{T}}^{2}+2 p_{\mathrm{T}}
$$

Note that (8) and 9) are (strictly) concave over the feasible region. Thus, differentiating them with respect to $p_{\mathrm{T}}$, and setting the derivatives to 0 , we find the optimal transmission probability $p_{\mathrm{T}}^{*}$ which maximizes the expected number of successful receptions in each slot as

$$
p_{\mathrm{T}}^{*}= \begin{cases}\frac{\tau^{\frac{2}{\eta}}+2-\sqrt{\left(\tau^{\frac{2}{\eta}}-1\right)^{2}+3}}{3 \tau^{\frac{2}{\eta}}}, & \text { if } \quad \tau<1 \\ \frac{\tau^{-\frac{2}{\eta}}-4+\sqrt{\left(\tau^{-\frac{2}{\eta}}-1\right)^{2}+3}}{3\left(\tau^{-\frac{2}{\eta}}-2\right)}, & \text { if } \quad \tau \geq 1\end{cases}
$$

In Fig. 11 we plot 10 as a function of $\tau$ with path loss exponent $\eta=4$. It can be seen that as threshold $\tau$ increases, $p_{\mathrm{T}}^{*}$ decreases. This is because at a higher threshold, it becomes more difficult for multiple transmitters to simultaneously satisfy the SINR criterion. We can also see that the maximum expected number of successful receptions $E\left[\left|\mathbf{I}_{t}^{s}\right|\right]^{*}$ decreases as the threshold $\tau$ increases. However, it does not necessarily mean that we will have a reduced number of successful receptions in a unit time, because the transmission duration will also become shorter. We will look at this issue in the subsequent section.

Remark 4.1: In [1], the optimal transmission probability under the collision channel model was derived, and was given by the inverse of the total number of nodes as in the original slotted ALOHA system. Recall that as $\tau$ goes to $\infty$, our 


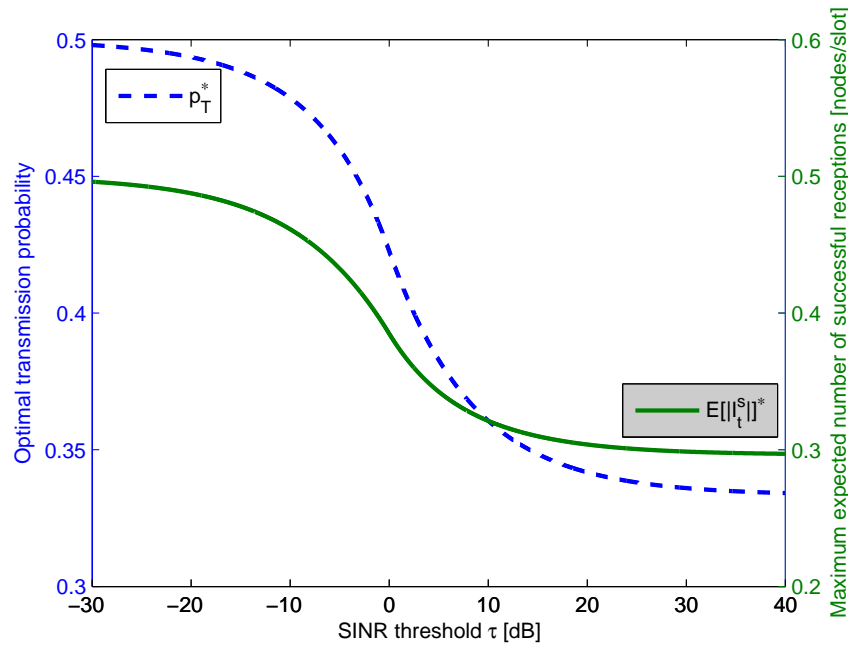

Fig. 1. Optimal transmission probability $p_{\mathrm{T}}^{*}$ and corresponding maximum expected number of successful receptions $E\left[\left|\mathbf{I}_{t}^{s}\right|\right]^{*}$ for the three node example

model accommodates the collision channel model. In (10), we have $p_{\mathrm{T}}^{*}=1 / 3$ as $\tau$ goes to $\infty$, which is consistent with the quoted result. Therefore, we conclude that the optimal transmission probability derived under collision channel model is fundamentally assuming the worst prior on the channel conditions by neglecting all the possibilities of succeeding in the presence of interference.

\section{B. On the Effect of the SINR Threshold}

For a given modulation scheme and target BER, the data rate is an increasing function of the SINR threshold $\tau$ [18]. Hence, if the number of bits to be transmitted is fixed and we increase $\tau$, the slot duration must be shortened due to the increased rate. However, as we have seen in the previous section, the transmission probability $p_{\mathrm{T}}$ needs to be lowered. To investigate this interrelationship, let us consider the $M$-PSK modulation scheme 2 in which its symbol rate [symbols/sec/Hz] at a given target BER $z$ is given by

$$
R_{s} \approx \begin{cases}\min \left\{\frac{2 \tau}{\left[Q^{-1}(z)\right]^{2}}, R_{s}^{\mathrm{m}}\right\}, & M=2(\mathrm{BPSK}) \\ \min \left\{\frac{\tau}{2\left[Q^{-1}(z)\right]^{2}}, R_{s}^{\mathrm{m}}\right\}, & M=4(\mathrm{QPSK}) \\ \min \left\{\frac{2 \tau \sin ^{2}(\pi / M)}{\left[Q^{-1}\left(\frac{z \log _{2} M}{2}\right)\right]^{2}}, R_{s}^{\mathrm{m}}\right\}, & M=2^{n}, n>2\end{cases}
$$

where $Q(z)$ is the probability that the standard normal random variable is greater than $z$. The maximum symbol rate $R_{s}^{\mathrm{m}}$ is given by $R_{s}^{\mathrm{m}}=1 / k_{g}$, where $k_{g}$ is the constant that depends on the pulse shape of the analog signal. Without loss of generality, we set $k_{g}=1$ (i.e., the raised cosine pulse with roll-off factor of 1). Denote by $W$ the number of bits to be transmitted in each time slot. Then, the transmission duration is given by $T_{\text {slot }}=\frac{W}{R_{s} B \log _{2} M}$. Consequently, we can redraw the maximum expected number of successful receptions as in Fig. 2, where we set $\eta=4, z=10^{-6}, W=1$ bit, and

\footnotetext{
${ }^{2}$ We could use the Shannon capacity formula but it would not make practical sense since it gives an asymptotic limit of the rate with arbitrarily small probability of error and arbitrarily long block length.
}

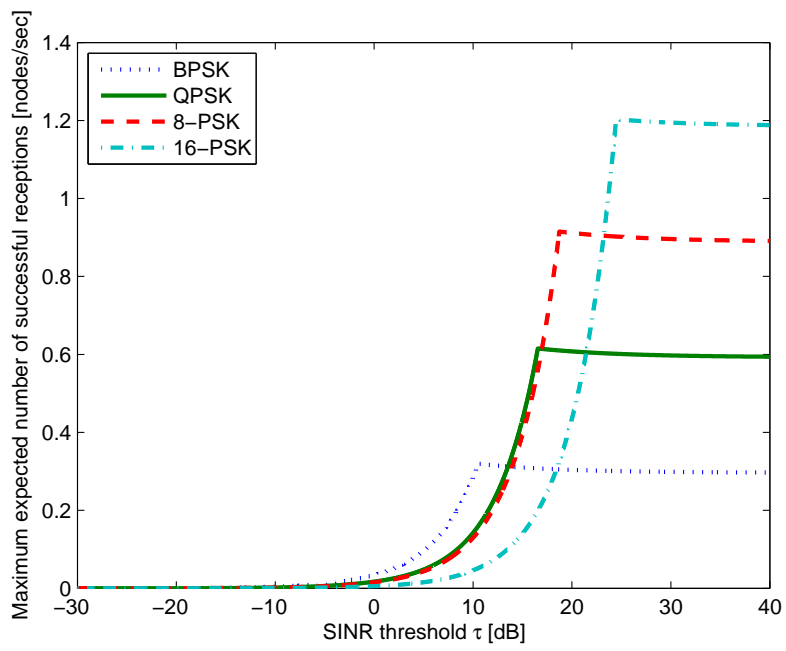

Fig. 2. The maximum expected number of successful receptions per second under the $M$-PSK modulation scheme

$B=1 \mathrm{~Hz}$, respectively. Note that in the figure, the units were changed from [nodes/slot] to [nodes/sec].

\section{Performance over Multiple Slots}

Because as time goes on, the set of discovered neighbors in each time slot will overlap with the previously discovered ones, it is important to know the performance of the discovery algorithm over multiple slots. We start with a simple scenario in which the set of successful transmitters $\mathbf{I}_{t}^{s}$ is independent from slot to slot. This is an artificial scenario because the set $\mathbf{I}_{t}^{s}$ is correlated in the sense that a node closer to the receiver has a better chance of being discovered in any slots. Therefore, the scenario where $\mathbf{I}_{t}^{s}$ is correlated over slots will also be studied later in this section.

Assuming that the set of successful transmitters $\mathbf{I}_{t}^{s}$ is independent from slot to slot implies that each neighboring node has equal probability of success. Therefore, for a given number of successful receptions $h_{t}$ and the total number of neighbors $J$, the probability that a certain neighboring node belongs to the set $\mathbf{I}_{t}^{s}$ is given by $h_{t} / J$ and, over $D$ multiple slots, the probability is obtained by

$$
\begin{aligned}
\operatorname{Pr}\left\{k \in \bigcup_{t=1}^{D} \mathbf{I}_{t}^{s}|| \mathbf{I}_{1}^{s} \mid=h_{1}, \ldots,\right. & \left.\left|\mathbf{I}_{D}^{s}\right|=h_{D}\right\} \\
& =1-\prod_{t=1}^{D}\left(1-\frac{h_{t}}{J}\right)
\end{aligned}
$$

We call 111 a slot-basis prediction to distinguish from the Bernoulli approximation which will be given in the sequel.

Because the event that a particular neighboring node belongs to the set $\mathbf{I}_{t}^{s}$ can be approximated by the Bernoulli trial with success probability $E\left[\left|\mathbf{I}_{t}^{s}\right|\right] / J$, the number of discovering the particular node over $D$ multiple slots is a binomial random variable with success probability $E\left[\left|\mathbf{I}_{t}^{s}\right|\right] / J$ and the total number of trials $D$. For large $D$ and small $E\left[\left|\mathbf{I}_{t}^{s}\right|\right] / J$, it can be further approximated by the Poisson random variable with parameter $D E\left[\left|\mathbf{I}_{t}^{s}\right|\right] / J$ [19, p. 435]. Hence, the probability 


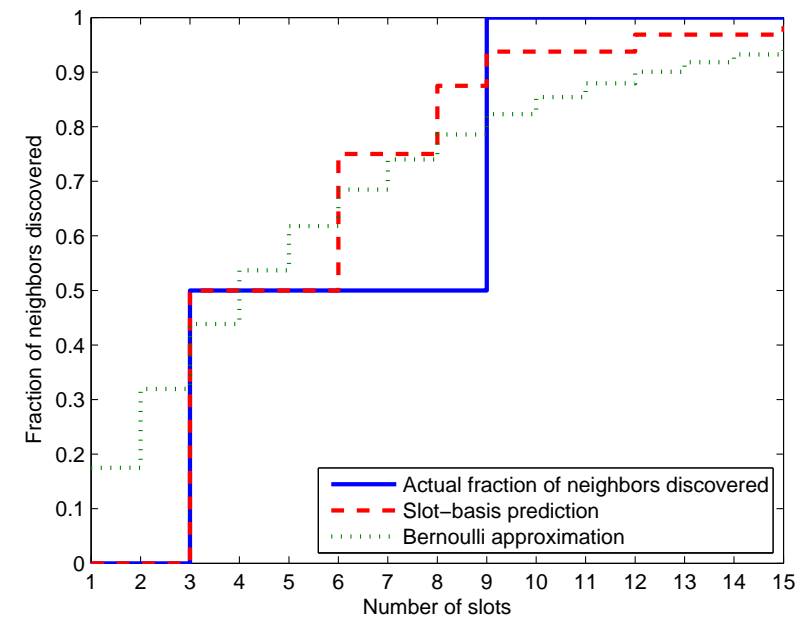

Fig. 3. The fraction of neighbors discovered by node $A$ for the example transmit/listen pattern in Table I

TABLE I

AN EXAMPLE TRANSMIT/LISTEN PATTERN OF THREE NODES (THE LETTERS T AND L STAND FOR TRANSMIT AND LISTEN, RESPECTIVELY.)

\begin{tabular}{c||ccccccccccccccc}
\hline Time slot & 1 & 2 & 3 & 4 & 5 & 6 & 7 & 8 & 9 & 10 & 11 & 12 & 13 & 14 & 15 \\
\hline \hline Node $A$ & T & T & L & L & T & L & T & L & L & T & T & L & T & T & L \\
\hline Node $B$ & L & L & T & L & T & T & L & T & L & L & L & L & T & T & T \\
\hline Node $C$ & L & T & T & L & T & L & L & T & T & T & T & T & T & T & $\mathrm{L}$ \\
\hline
\end{tabular}

that the particular node has been discovered over $D$ multiple slots is approximately $1-\exp \left(-D E\left[\left|\mathbf{I}_{t}^{s}\right|\right] / J\right)$, which is equal to the probability that the Poisson random variable is nonzero. Note that the slot-basis prediction in (11) and the above approximation do not depend on the particular node index because we assumed that $\mathbf{I}_{t}^{s}$ is independent from slot to slot. Therefore, it can be viewed as the predicted fraction of neighbors discovered up to time slot $D$. In Fig. 3 , we plot the actual/predicted fraction of neighbors discovered for the example transmit/listen pattern in Table [] For the figure, the SINR threshold $\tau$ is set to 1 (in linear scale), and the transmission probability $p_{\mathrm{T}}$ is set to 0.4226 which is the optimal value by (10) with path loss exponent $\eta=4$. Note that this is the optimal transmission probability which maximizes the expected number of successful receptions at that threshold value. Consequently, we obtain the expected number of successful receptions per slot as 0.3849 by (9), and this value was used to plot the Bernoulli approximation.

In practice, the probability that a particular node belongs to the set of successful transmitters $\mathbf{I}_{t}^{s}$ depends on the distance between the node and the receiver and, thus, $\mathbf{I}_{t}^{s}$ is correlated over time slots. Therefore, we first obtain the conditional probability that a particular node at the specific distance from the receiver belongs to the set $\mathbf{I}_{t}^{s}$ given the total number of successful receptions $h_{t}$ as (see Appendix B)

$$
\begin{aligned}
& \operatorname{Pr}\left\{k \in \mathbf{I}_{t}^{s}|| \mathbf{I}_{t}^{s} \mid=h_{t}, r_{k}=r^{\prime}\right\} \\
& =\frac{\sum_{n=h_{t}}^{J}\left(\begin{array}{l}
J-1 \\
n-1
\end{array}\right) p_{\mathrm{T}}^{n}\left(1-p_{\mathrm{T}}\right)^{J-n} \zeta_{n}}{\sum_{n=h_{t}}^{J}\left(\begin{array}{l}
J \\
n
\end{array}\right) p_{\mathrm{T}}^{n}\left(1-p_{\mathrm{T}}\right)^{J-n}\left(\frac{n}{J}\left(\zeta_{n}+\gamma_{n}-\xi_{n}\right)+\xi_{n}\right)}
\end{aligned}
$$

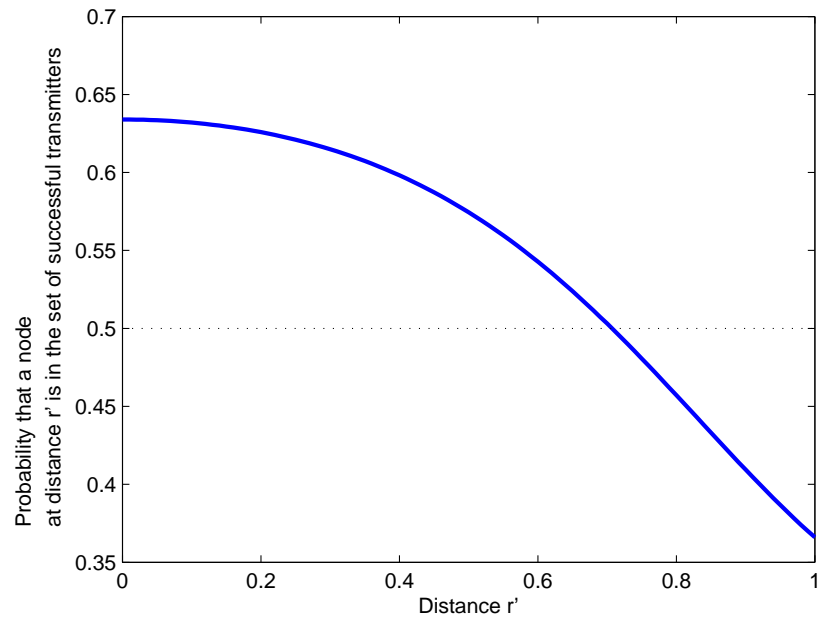

Fig. 4. The probability that a node at distance $r^{\prime}$ from the reference node is in the set of successful transmitters for the three node example given the number of successful transmissions $h_{t}=1$

where $\zeta_{n}, \gamma_{n}$, and $\xi_{n}$ are defined as

$$
\begin{aligned}
\zeta_{n} & =\left(\begin{array}{c}
n-1 \\
h_{t}-1
\end{array}\right) f_{1, n} f_{2, n}^{h_{t}-1}\left(1-f_{2, n}\right)^{n-h_{t}} \\
\gamma_{n} & =\left(\begin{array}{c}
n-1 \\
h_{t}
\end{array}\right) f_{2, n}^{h_{t}}\left(1-f_{2, n}\right)^{n-h_{t}-1}\left(1-f_{1, n}\right) \\
\xi_{n} & =\left(\begin{array}{c}
n \\
h_{t}
\end{array}\right) f_{n}^{h_{t}}\left(1-f_{n}\right)^{n-h_{t}}
\end{aligned}
$$

and $f_{1, n}$ is the probability that a transmitter located at distance $r^{\prime}$ from the receiver will succeed among $n(\geq 1)$ simultaneous transmissions and $f_{2, n}$ is the probability that a transmitter at an arbitrary distance will succeed among $n(>1)$ simultaneous transmissions given that one of the other transmitter is located at distance $r^{\prime}$ from the receiver. The expressions for $f_{1, n}$ and $f_{2, n}$ are given in Appendix $\mathrm{B}$. The function $f_{n}$ is the shorthand notation for (7). Using the conditional probability in (12), the probability that a particular node at distance $r^{\prime}$ has been discovered over multiple slots can be obtained similarly with (11). To get a better understanding on the conditional probability (12), let us consider the following example which is in line with the Example 4.1 .

Example 4.2: Since each node has two neighbors, it is obvious that $\operatorname{Pr}\left\{k \in \mathbf{I}_{t}^{s}|| \mathbf{I}_{t}^{s} \mid=0, r_{k}=r^{\prime}\right\}=0$ and $\operatorname{Pr}\left\{k \in \mathbf{I}_{t}^{s}|| \mathbf{I}_{t}^{s} \mid=2, r_{k}=r^{\prime}\right\}=1$. For $h_{t}=1$, after some calculation, we obtain

$$
\begin{aligned}
\operatorname{Pr}\left\{k \in \mathbf{I}_{t}^{s}|| \mathbf{I}_{t}^{s} \mid=1, r_{k}=r^{\prime}\right\} \\
=\frac{1-p_{\mathrm{T}}+p_{\mathrm{T}}\left(1-{r^{\prime}}^{2}\right)^{2}}{2\left(1-p_{\mathrm{T}}\right)+\left(\left(1-{r^{\prime}}^{2}\right)^{2}+{r^{\prime}}^{4}\right) p_{\mathrm{T}}}
\end{aligned}
$$

where the discovery range $R_{0}$ in (1) is normalized to 1 and the threshold $\tau$ is set to 1 . We plot the result in Fig. 4 where all other parameters are set identical with those used for Fig 3 Note that even if $r^{\prime}=0$, the probability is not equal to 1 because of the randomness on node's transmission. 


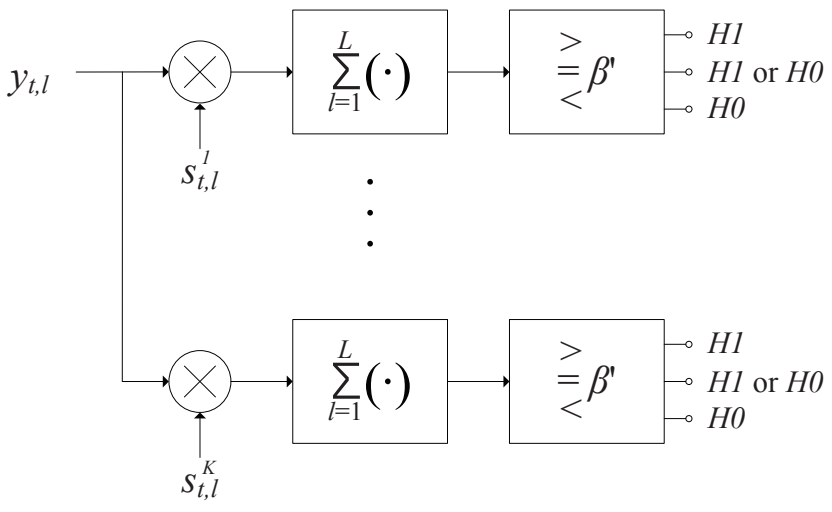

Fig. 5. Bank of matched filters

\section{Physical Layer Signal Processing: Detection OF the TransmitTing NeIghbors}

\section{A. Classical Approach Using a Bank of Matched Filters}

To decide the existence of a signal from a particular node, we require the use of matched filters in which the outputs of the filters are compared to a certain threshold. Note that such a decision is subject to probabilistic errors such as the false alarm and miss and, thus, the threshold needs to be chosen in some optimum way. Since the set of transmitting neighbors and their signal amplitudes are all unknown and hard to be tracked, we simplify it by assuming that the sum of interfering signals and the noise act as another noise process $\mathbf{n}_{t}^{\prime}=\left\{n_{t, 1}^{\prime}, \ldots, n_{t, L}^{\prime}\right\}^{T}$ whose samples are i.i.d. with $\mathcal{N}\left(0, N^{\prime}\right)$. The average noise power $N^{\prime}$ can be computed as

$$
N^{\prime}=N+\sum_{n=1}^{\bar{J}-1}\left(\begin{array}{c}
\bar{J}-1 \\
n
\end{array}\right) p_{\mathrm{T}}^{n}\left(1-p_{\mathrm{T}}\right)^{\bar{J}-1-n} n \bar{P}_{r x}
$$

where $\bar{J}$ and $\bar{P}_{r x}$ is the average number of neighbors and the received signal power, respectively. Note that this is a reasonable approximation if there is a large number of nodes, and it is commonly applied to the analysis of cellular code division multiple access (CDMA) systems [18].

Based on the above approximation, we formulate the binary hypothesis testing problem for the transmission of a particular node $k$ as

$$
\begin{aligned}
& H_{0}: \mathbf{y}_{t}=\mathbf{n}_{t}^{\prime} \\
& \text { versus } \\
& H_{1}: \mathbf{y}_{t}=g_{t}^{k} \mathbf{s}_{t}^{k}+\mathbf{n}_{t}^{\prime}
\end{aligned}
$$

which is a composite hypothesis testing because the signal $\mathbf{s}_{t}^{k}$ is known but still not its amplitude. For the problem, a generalized likelihood ratio test method would provide a simple decision rule as 320 , p. 51]

$$
\frac{1}{N^{\prime}} \sum_{l=1}^{L} y_{t, l} s_{t, l}^{k} \underset{H_{0}}{\stackrel{H_{1}}{\gtrless}} \beta
$$

This structure is depicted in Fig. 5, where $\beta^{\prime}=\beta N^{\prime}$. Note that each node transmits with probability $p_{\mathrm{T}}$ and listens with

\footnotetext{
${ }^{3}$ Note that the decision rule in 19 is optimal for $g_{t}^{k}$ near 0 which holds for most of the practical situations.
}

probability $1-p_{\mathrm{T}}$, and they are the prior probabilities for each hypothesis.

Given the cost structure $C_{i j}$ which is the cost incurred by choosing hypothesis $H_{i}$ when hypothesis $H_{j}$ is true, the optimum threshold for the minimum average cost is given by $\beta=\frac{\pi_{0}\left(C_{10}-C_{00}\right)}{\pi_{1}\left(C_{01}-C_{11}\right)}$, where $\pi_{i}$ is the prior probability of hypothesis $H_{i}$. Under the minimum-probability-of-error criterion where the cost assignment is done by $C_{i j}=0$ for $i=j$, and $C_{i j}=1$ for $i \neq j$, the threshold is obtained by $\beta=\pi_{0} / \pi_{1}=\left(1-p_{\mathrm{T}}\right) / p_{\mathrm{T}}$.

\section{B. Random Set Theory-based Approach}

1) Motivation and Background: The classical approach using a bank of matched filters holds a certain desired property; that is, its complexity does not scale with the number of nodes by averaging out the effect of random interferences. However, the performance would be worse than the class of decorrelator detectors. On the other hand, total number of transmitting neighbors and their entities are all random in our problem setting and, thus, standard decorrelator detectors are not directly applicable because they fundamentally assume a fixed number of transmitters with known entities. This problem naturally falls in the purview of RST which generalizes standard probability theory by assigning sets, rather than values, to random outcomes (see Appendix $\AA$ and references therein). RST has been applied before in the context of multi-source data fusion and multi-target identification problems [10]-[15] and, recently, multi-user detection problem in a dynamic environment [16]. The utility of RST mostly comes from the fact that we can readily treat the random behavior of a random number of entities as a single random set having likelihood.

Mathematically, a random set $\mathbf{X}$ is defined as a mapping from a sample space $\Omega$ to a power set $\mathcal{P}(\mathbb{S})$ of a hybrid space $\mathbb{S}$. It is referred to as a random finite set, if for all $\omega \in \Omega$, the set is finite (i.e., $|\mathbf{X}(\omega)|<\infty$ ). The hybrid space $\mathbb{S} \triangleq \mathbb{R}^{d} \times U$ is the Cartesian product of a $d$-dimensional Euclidean space $\mathbb{R}^{d}$ and a finite discrete space $U$. To illustrate the use of the RST-based method, we focus on the case where the hybrid space $\mathbb{S}$ is given by a finite discrete space as $\mathbb{S}=\{1, \ldots, K\}$, i.e., the random set $\mathbf{X}_{t}$ is simply equal to the unknown set of transmitting neighbors at that time slot, which was previously denoted by $\mathbf{I}_{t}$ in (2). Additionally, if the amplitudes of the signals from transmitting neighbors are of interest, we can defined it as $\mathbb{S}=\{1, \ldots, K\} \times \mathbb{R}_{+}$, where $\mathbb{R}_{+}$is the nonnegative real space. In the following, we estimate the random set $\mathbf{X}_{t}$ based on the observed signal $\mathbf{y}_{t}$ in each time slot.

2) Defining the Estimator: Since the number of transmitting neighbors in each time slot and the total number of neighbors are connected, both the random set $\mathbf{X}_{t}$ and the total number of neighbors $J$ need to be jointly estimated as

$$
\arg \max _{\left(\mathbf{X}_{t}^{\prime}, J^{\prime}\right)} f_{\mathbf{X}_{t}, J \mid \mathbf{Y}_{t}}\left(\mathbf{X}_{t}^{\prime}, J^{\prime} \mid \mathbf{y}_{t}\right)
$$

where $f_{\mathbf{X}_{t}, J \mid \mathbf{Y}_{t}}(\cdot)$ is the likelihood of random set $\mathbf{X}_{t}$ and $J$ neighbors given the received signal $\mathbf{Y}_{t}=\mathbf{y}_{t}$. By Bayes rule, $f_{\mathbf{X}_{t}, J \mid \mathbf{Y}_{t}}\left(\mathbf{X}_{t}^{\prime}, J^{\prime} \mid \mathbf{y}_{t}\right)$ is proportional to

$$
f_{\mathbf{Y}_{t} \mid \mathbf{X}_{t}, J}\left(\mathbf{y}_{t} \mid \mathbf{X}_{t}^{\prime}, J^{\prime}\right) f_{\mathbf{X}_{t} \mid J}\left(\mathbf{X}_{t}^{\prime} \mid J^{\prime}\right) f_{J}\left(J^{\prime}\right)
$$


where $f_{\mathbf{Y}_{t} \mid \mathbf{X}_{t}, J}(\cdot)$ is the likelihood of received signal $\mathbf{Y}_{t}$ given $\mathbf{X}_{t}=\mathbf{X}_{t}^{\prime}$ and $J=J^{\prime}, f_{\mathbf{X}_{t} \mid J}(\cdot)$ is the likelihood of random set $\mathbf{X}_{t}$ given $J=J^{\prime}$, and $f_{J}(\cdot)$ is the likelihood of having $J$ neighbors. Consequently, the joint MAP estimator of the random set $\mathbf{X}_{t}$ and the total number of neighbors $J$ is obtained by

$$
\arg \max _{\left(\mathbf{X}_{t}^{\prime}, J^{\prime}\right)} f_{\mathbf{Y}_{t} \mid \mathbf{X}_{t}, J}\left(\mathbf{y}_{t} \mid \mathbf{X}_{t}^{\prime}, J^{\prime}\right) f_{\mathbf{X}_{t} \mid J}\left(\mathbf{X}_{t}^{\prime} \mid J^{\prime}\right) f_{J}\left(J^{\prime}\right)
$$

In order to run the above estimator, it is required to specify the densities $f_{\mathbf{Y}_{t} \mid \mathbf{X}_{t}, J}(\cdot), f_{\mathbf{X}_{t} \mid J}(\cdot)$, and $f_{J}(\cdot)$. We outline how these densities are obtained.

3) Specifying the Densities: The received signal $\mathbf{y}_{t}$ not only depends on the set of transmitting neighbors $\mathbf{X}_{t}$, but also depends on their signal amplitudes $\mathbf{g}_{t}=\left\{g_{t}^{k}, \forall k \in \mathbf{X}_{t}\right\}$. Since the amplitudes are all random, we take average by assuming that the amplitudes of the nodes are independent of each other as

$$
\begin{aligned}
& f_{\mathbf{Y}_{t} \mid \mathbf{X}_{t}, J}\left(\mathbf{y}_{t} \mid \mathbf{X}_{t}^{\prime}, J^{\prime}\right) \\
& =\int_{0}^{\infty} \cdots \int_{0}^{\infty} f_{\mathbf{Y}_{t} \mid \mathbf{X}_{t}, \mathbf{g}_{t}, J}\left(\mathbf{y}_{t} \mid \mathbf{X}_{t}^{\prime}, \mathbf{g}_{t}^{\prime}, J^{\prime}\right) d F_{g}\left(g_{1}^{\prime}\right) \cdots d F_{g}\left(g_{\left|\mathbf{X}_{t}^{\prime}\right|}^{\prime}\right)
\end{aligned}
$$

where $f_{\mathbf{Y}_{t} \mid \mathbf{X}_{t}, \mathbf{g}_{t}, J}\left(\mathbf{y}_{t} \mid \mathbf{X}_{t}^{\prime}, \mathbf{g}_{t}^{\prime}, J^{\prime}\right)$ is the likelihood of received signal $\mathbf{Y}_{t}$ given $\mathbf{X}_{t}=\mathbf{X}_{t}^{\prime}, \mathbf{g}_{t}=\mathbf{g}_{t}^{\prime}$, and $J=J^{\prime}$. The set $\mathbf{g}_{t}^{\prime}=\left\{g_{1}^{\prime}, \ldots, g_{\mid \mathbf{X}_{t}^{\prime}}^{\prime}\right\}$ and $F_{g}(\cdot)$ denote the realization of $\mathbf{g}_{t}$ and the common cumulative distribution of the received signal amplitude, respectively. Note that by further conditioning the received signal $\mathbf{y}_{t}$ on the set of transmitting neighbors and their signal amplitudes, the only randomness remaining is in the noise $\mathbf{n}_{t}$. Therefore, it is given by

$$
\begin{aligned}
& f_{\mathbf{Y}_{t} \mid \mathbf{X}_{t}, \mathbf{g}_{t}, J}\left(\mathbf{y}_{t} \mid \mathbf{X}_{t}^{\prime}, \mathbf{g}_{t}^{\prime}, J^{\prime}\right) \\
& =\frac{1}{(2 \pi N)^{L / 2}} \exp \left(-\frac{1}{2 N} \sum_{l=0}^{L}\left(y_{t, l}-\sum_{k \in \mathbf{X}_{t}^{\prime}} g_{m(k)}^{\prime} s_{t, l}^{k}\right)^{2}\right)
\end{aligned}
$$

where the bijective function $m(k)$ has been introduced to map the elements in $\mathbf{X}_{t}^{\prime}$ to the elements in the set $\left\{1, \ldots,\left|\mathbf{X}_{t}^{\prime}\right|\right\}$. For example, if $\mathbf{X}_{t}^{\prime}=\{2,5\}$, then $m(2)=1$ and $m(5)=2$.

In order to obtain $f_{\mathbf{X}_{t} \mid J}\left(\mathbf{X}_{t}^{\prime} \mid J^{\prime}\right)$, we first obtain the belief mass of a random set $\mathbf{X}_{t}$ for a given number of neighbors $J=J^{\prime}$ as (see Appendix $\mathrm{A}$ )

$$
\beta_{\mathbf{X}_{t} \mid J}\left(\mathbf{C} \mid J^{\prime}\right)=\sum_{n=0}^{J^{\prime}} \sum_{\mathbf{B}: \mathbf{B} \subseteq \mathbf{C},|\mathbf{B}|=n} \operatorname{Pr}\left\{\mathbf{X}_{t}=\mathbf{B} \mid J=J^{\prime}\right\}
$$

where $\mathbf{C}$ is a closed subset of the space $\mathbb{S}$, and $\mathbf{B}$ is a realization of the random set $\mathbf{X}_{t}$. Let us first derive $\beta_{\mathbf{X}_{t} \mid J}\left(\mathbf{C} \mid J^{\prime}\right)$ for a particular example as follows.

Example 5.1: Set $\mathbb{S}=\{1,2,3\}, \mathbf{C}=\mathbb{S}$, and $J^{\prime}=2.1$ ) The set $\{\mathbf{B}: \mathbf{B} \subseteq \mathbf{C},|\mathbf{B}|=0\}$ is given by $\{\emptyset\}$ and, thus, $\sum_{\mathbf{B}: \mathbf{B} \subseteq \mathbf{C},|\mathbf{B}|=0} \operatorname{Pr}\left\{\mathbf{X}_{t}=\mathbf{B} \mid J=2\right\}=\left(1-p_{\mathrm{T}}\right)^{2}$. 2) The set $\{\mathbf{B}: \mathbf{B} \subseteq \mathbf{C},|\mathbf{B}|=1\}$ is given by $\{\{1\},\{2\},\{3\}\}$ and, thus, $\sum_{\mathbf{B}: \mathbf{B} \subseteq \mathbf{C},|\mathbf{B}|=1} \operatorname{Pr}\left\{\mathbf{X}_{t}=\mathbf{B} \mid J=2\right\}=3 p_{\mathrm{T}}\left(1-p_{\mathrm{T}}\right)$. 3) The set $\{\mathbf{B}: \mathbf{B} \subseteq \mathbf{C},|\mathbf{B}|=2\}$ is given by $\{\{1,2\},\{1,3\},\{2,3\}\}$ and, thus, $\sum_{\mathbf{B}: \mathbf{B} \subseteq \mathbf{C},|\mathbf{B}|=2} \operatorname{Pr}\left\{\mathbf{X}_{t}=\mathbf{B} \mid J=2\right\}=3 p_{\mathrm{T}}{ }^{2}$. 4) Since $J^{\prime}=2$, the probability that a random set $\mathbf{X}_{t}$ is equal to
$\mathbf{B}$ for $|\mathbf{B}|>2$ is zero. Summing over all possible B's yields $\beta_{\mathbf{X}_{t} \mid J}\left(\mathbf{C} \mid J^{\prime}\right)=\sum_{n=0}^{2}\left(\begin{array}{l}3 \\ n\end{array}\right) p_{\mathrm{T}}^{n}\left(1-p_{\mathrm{T}}\right)^{2-n}$.

For general cases, we have

$$
\beta_{\mathbf{X}_{t} \mid J}\left(\mathbf{C} \mid J^{\prime}\right)=\sum_{n=0}^{J^{\prime}}\left(\begin{array}{c}
|\mathbf{C}| \\
n
\end{array}\right) p_{\mathrm{T}}^{n}\left(1-p_{\mathrm{T}}\right)^{J^{\prime}-n}
$$

The belief density $f_{\mathbf{X}_{t} \mid J}\left(\mathbf{X}_{t}^{\prime} \mid J^{\prime}\right)$ is obtained by taking the set derivative of the belief mass obtained above. For the case where the hybrid space $\mathbb{S}$ is comprised only of the discrete space, it can be readily obtained through the following Möbius inversion formula as (see Appendix A)

$$
f_{\mathbf{X}_{t} \mid J}\left(\mathbf{X}_{t}^{\prime} \mid J^{\prime}\right)=\sum_{\mathbf{C} \subseteq \mathbf{X}_{t}^{\prime}}(-1)^{\left|\mathbf{X}_{t}^{\prime} \backslash \mathbf{C}\right|} \beta_{\mathbf{X}_{t} \mid J}\left(\mathbf{C} \mid J^{\prime}\right)
$$

Example 5.2: Take $\mathbf{X}_{t}^{\prime}=\{1,3\}$ and $J^{\prime}=2$, then the set $\left\{\mathbf{C}: \mathbf{C} \subseteq \mathbf{X}_{t}^{\prime}\right\}$ is given by $\{\emptyset,\{1\},\{3\},\{1,3\}\}$. 1) For $\mathbf{C}=\emptyset, \beta_{\mathbf{X}_{t} \mid J}\left(\mathbf{C} \mid J^{\prime}\right)=\left(1-p_{\mathrm{T}}\right)^{2}$. 2) For $\mathbf{C}=\{1\}$, $\left.\beta_{\mathbf{X}_{t} \mid J}\left(\mathbf{C} \mid J^{\prime}\right)=\left(1-p_{\mathrm{T}}\right)^{2}+p_{\mathrm{T}}\left(1-p_{\mathrm{T}}\right) .3\right)$ For $\mathbf{C}=\{3\}$, $\left.\beta_{\mathbf{X}_{t} \mid J}\left(\mathbf{C} \mid J^{\prime}\right)=\left(1-p_{\mathrm{T}}\right)^{2}+p_{\mathrm{T}}\left(1-p_{\mathrm{T}}\right) .4\right)$ For $\mathbf{C}=\{1,3\}$, $\beta_{\mathbf{X}_{t} \mid J}\left(\mathbf{C} \mid J^{\prime}\right)=\left(1-p_{\mathrm{T}}\right)^{2}+2 p_{\mathrm{T}}\left(1-p_{\mathrm{T}}\right)+p_{\mathrm{T}}{ }^{2}$. Summing over all the possible C's by considering the sign of the terms yields $f_{\mathbf{X}_{t} \mid J}\left(\mathbf{X}_{t}^{\prime} \mid J^{\prime}\right)=p_{\mathrm{T}}{ }^{2}$.

Similarly, for general cases, we have

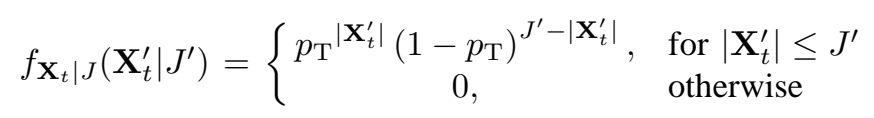

Note that the scenario in which $\mathbf{X}_{t}$ contains only the identity of the transmitters is the simplest case that can be solved by standard probability theory as well. The usefulness of RST comes when we extend the set $\mathbf{X}_{t}$ so that additional parameters, such as signal amplitudes, can be estimated at the same time.

The density $f_{J}\left(J^{\prime}\right)$ is the probability that there are $J^{\prime}$ number of neighbors. By defining the discovery region as the circle of radius $R_{0}$, from Section W the number of nodes inside the discovery region follows a Poisson random variable with parameter $\lambda \pi R_{0}{ }^{2}$. Notice that there is no definite way of choosing $R_{0}$ because the decisions on whether a particular node is my neighbor are inconclusive due to the continuity of signal strength together with the random effect of noise and fading. However, as will be shown in the numerical example, the estimator gives more weights to an appropriate size of the set which is likely to be occurred at that size of discovery region.

\section{NumericAl RESULtS}

Since comparing the performance of the discovery algorithm under collision channel to that under multipacket reception channel is trivial and obvious, we focus on the comparison of the physical layer signal processing methods for the detection of transmitting neighbors. For the simulation, a total of 8 wireless sensor nodes are uniformly deployed over the region of interest which is modeled as a circle of radius $R$ which is set to $1 \mathrm{~km}$ as shown in Fig. 6. The reference node denoted by index 0 is assumed to be located at the center of the circle. For the wireless channel, we consider a simple path loss model 


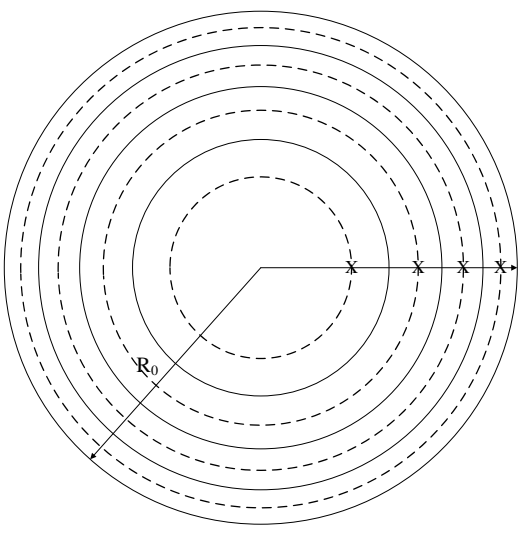

(a) Discretization of the distance

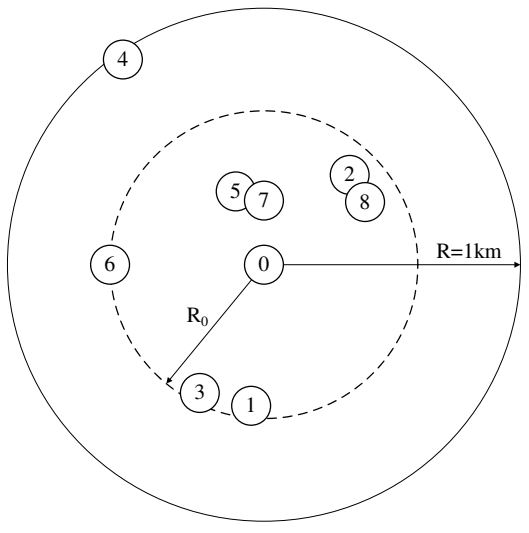

(b) Deployment scenario 1

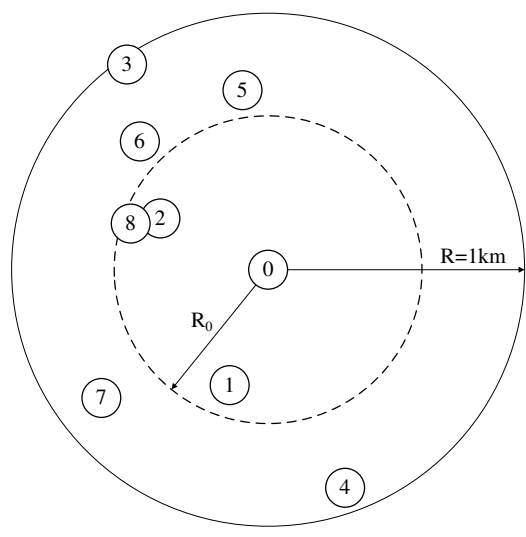

(c) Deployment scenario 2

Fig. 6. Discretization of the distance for fast computation of 21 and example deployment scenarios

which only depends on the transmitter-receiver distance, and the path loss exponent $\eta$ is set to 4 . The transmission power $G$ is set to $-24 \mathrm{dBm} 4$, and the noise spectral density $N_{0}$ is set to $-173 \mathrm{dBm} / \mathrm{Hz}$ as commonly done. The bandwidth $B$ is set to $100 \mathrm{~Hz}$ (which is artificially low but really irrelevant for our purposes here). The transmission probability $p_{\mathrm{T}}$ is set to 0.5 (to better observe the effect of the multiple access). For the signatures of the nodes, we used length 15 maximallength sequences [22]. Gold and Kasami sequences which have better correlation property can be used, but comparing the performance of different codes is beyond the scope of this paper.

Note that the computation of $f_{\mathbf{Y}_{t} \mid \mathbf{X}_{t}, J}(\cdot)$ in (21) is tedious because of the multiple integrals. Hence, we transform the integrals to a finite summation by discretizing the distance. To do this, we first divide the discovery region of the reference node into a finite number of strips having the same area and, after that, each strip is further divided into two having the same area as shown in Fig. 6(a). By doing so, the probability that a node is on either one of those radii becomes uniform. For the numerical examples, we used 7 discrete points and, obviously, the accuracy will improve as the number of points increases. Also note that, since a total of 8 nodes are uniformly deployed over the specified region of interest, the density $f_{J}(\cdot)$ on the number of nodes inside the discovery region of radius $R_{0}$ can be more accurately described by the binomial distribution $B\left(8, \frac{R_{0}^{2}}{R^{2}}\right)$, rather than the Poisson approximation. For the classical approach, it is computed by $\beta^{\prime}=\beta N^{\prime}$ where $\beta=\left(1-p_{\mathrm{T}}\right) / p_{\mathrm{T}}=1$ and $N^{\prime}=N_{0} B+3.5 \bar{P}_{r x}$ by 117 with $\bar{P}_{r x}=3.3333 \times 10^{-7} G$ using the cumulative distribution function of the received signal power in (3) without fading.

Table [I and [II] show the detection results for deployment scenario 1 and 2 in Fig. 6(b). (c), respectively. The letters H, C, $\mathrm{F}$, and $\mathrm{M}$ stand for hit, correct rejection, false alarm, and miss, respectively. Note that $\mathrm{F}$ and $\mathrm{M}$ are the erroneous detections. From Table П it can be seen that a total of 1 miss and 3 false alarms are induced under the RST-based approach with $R_{0}=1 \mathrm{~km}$ (which is the entire region of interest), and 2

\footnotetext{
${ }^{4}$ For example, the transmission power of the MICAz Mote, a commercial wireless sensor node by Crossbow Technology, Inc., is programmable in 8 steps from -24 to $0 \mathrm{dBm}$ [21].
}

TABLE II

DETECTION RESULTS FOR DEPLOYMENT SCENARIO 1 IN FIG. 6(B) (THE LETTERS H, C, F, AND M STAND FOR hit, correct rejection, false alarm, AND miss, RESPECTIVELY.)

(a) RST-based approach $\left(R_{0}=1 \mathrm{~km}\right)$

\begin{tabular}{|c|c|}
\hline & $\begin{array}{llllllllllllllllllllll}1 & 2 & 3 & 4 & 5 & 6 & 7 & 8 & 9 & 10 & 11 & 12 & 13 & 14 & 15 & 16 & 17 & 18 & 19 & 20\end{array}$ \\
\hline Node 1 & 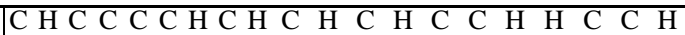 \\
\hline Node 2 & 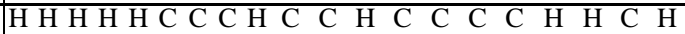 \\
\hline Node 3 & 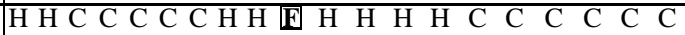 \\
\hline Node 4 & 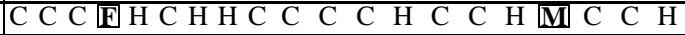 \\
\hline Node 5 & HCH H HCCC C C C \\
\hline Node 6 & 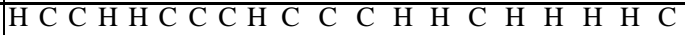 \\
\hline Node 7 & 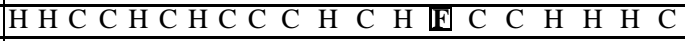 \\
\hline ode 8 & 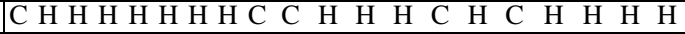 \\
\hline
\end{tabular}

(b) RST-based approach $\left(R_{0}=0.5 \mathrm{~km}\right)$

Time slot $\mid \begin{array}{llllllllllllllllll}1 & 2 & 3 & 4 & 5 & 6 & 7 & 8 & 9 & 10 & 11 & 12 & 1314 & 15 & 1617 & 18 & 19 & 20\end{array}$

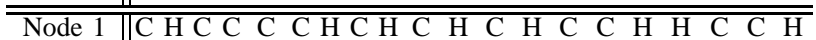

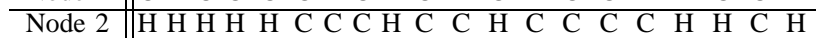

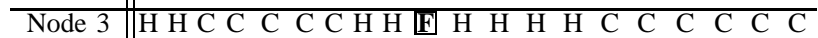

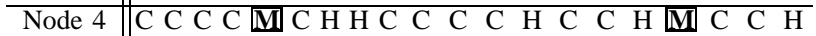

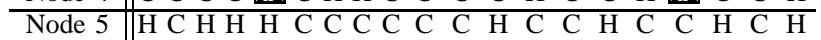

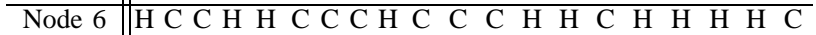
\begin{tabular}{l||lllllllllllll}
\hline Node 7 & H H C C H C H C C C & H C C H C C C C & H & H & H & C \\
\hline
\end{tabular}

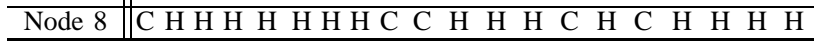

(c) Classical approach using a bank of matched filters

\begin{tabular}{|c|c|}
\hline & $\begin{array}{llllllllllllllllllllll}1 & 2 & 3 & 4 & 5 & 6 & 7 & 8 & 9 & 10 & 11 & 12 & 13 & 14 & 15 & 16 & 17 & 18 & 18\end{array}$ \\
\hline Node & 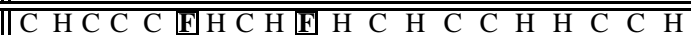 \\
\hline Node & 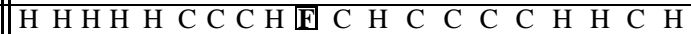 \\
\hline Node 3 & H H C C C C C H H \\
\hline Node 4 & 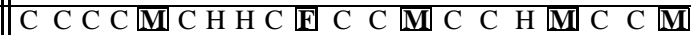 \\
\hline Node 5 & $\mathrm{HCHH} \mathrm{HCCCCCCHCCH}$ \\
\hline Node 6 & 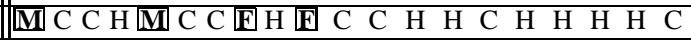 \\
\hline Node 7 & 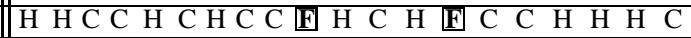 \\
\hline & \\
\hline
\end{tabular}

misses and 1 false alarm are occurred with $R_{0}=0.5 \mathrm{~km}$. Under the classical approach, however, a total of 6 misses and 10 false alarms occur, which is 4 times more than the RSTbased approach with $R_{0}=1 \mathrm{~km}$ and 5.3 times more than that with $R_{0}=0.5 \mathrm{~km}$.

From Table III, we can see that a total of 2 misses and 4 false alarms are induced under the RST-based approach with 
TABLE III

DETECTION RESULTS FOR DEPLOYMENT SCENARIO 2 IN FIG.6(C)

(a) RST-based approach $\left(R_{0}=1 \mathrm{~km}\right)$

Time slot $\mid \begin{array}{llllllllllllllllllll}1 & 2 & 3 & 4 & 5 & 6 & 7 & 8 & 9 & 10 & 11 & 12 & 13 & 14 & 15 & 16 & 17 & 18 & 19 & 20\end{array}$ \begin{tabular}{l|lllllllllllll}
\hline \hline Node 1 & C C C C C C H C H H C H H C H H H H C H & \\
\hline
\end{tabular}

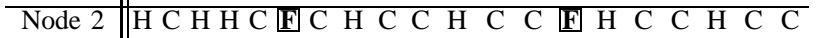

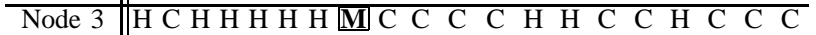

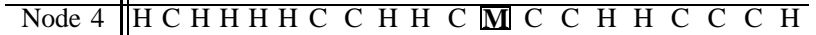
Node 5 H C C H H H $\mathbf{E}$ H H C H H C H C C H C H H

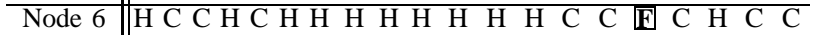
Node 7 C H C H H C C C H H H C C C H H H C C C

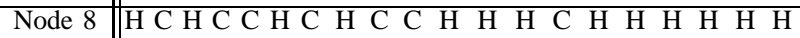

(b) RST-based approach $\left(R_{0}=0.5 \mathrm{~km}\right)$

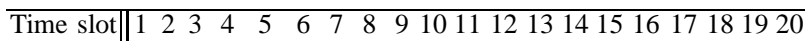
\begin{tabular}{l||lllllllllllllll}
\hline \hline Node 1 & C C C C C C C H C H H C H H C H H H H C H
\end{tabular}

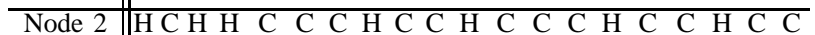

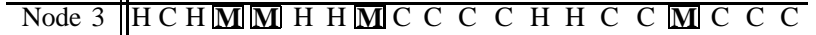

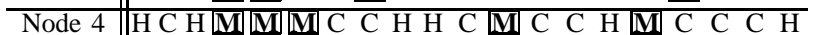

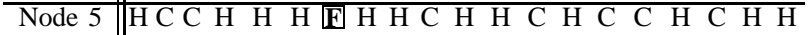
Node 6 H C C H C H H H H H H H H C C C C H C C

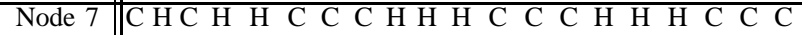
\begin{tabular}{l||lllllllllllllll}
\hline Node 8 & H C H C C H C H C C H H H C H H H H H H \\
\hline
\end{tabular}

(c) Classical approach using a bank of matched filters Time slot $\mid \begin{array}{llllllllllllllllllll}1 & 2 & 3 & 4 & 5 & 6 & 7 & 8 & 9 & 10 & 11 & 12 & 13 & 14 & 15 & 16 & 17 & 18 & 19 & 20\end{array}$ \begin{tabular}{l||lllllll}
\hline \hline Node 1 & C C C C C C H C H H C H H C H H H H C H \\
\hline
\end{tabular}

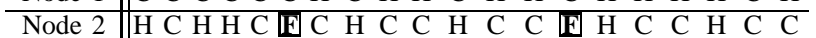

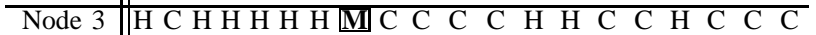

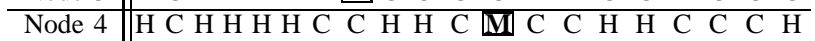

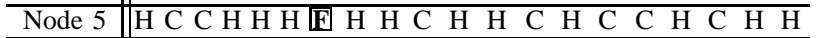

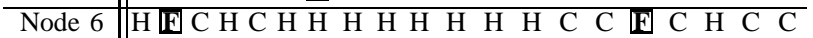

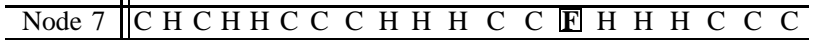

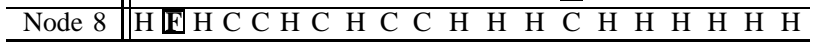

$R_{0}=1 \mathrm{~km}$, and 9 misses and 1 false alarm are occurred with $R_{0}=0.5 \mathrm{~km}$. Overall, by reducing the discovery range $R_{0}$, the occurrence of false alarm is reduced, whereas that of miss is increased. Note, however, that most of the misses are due to the nodes outside the discovery region (i.e., node 3 and 4). In fact, the detection of the nodes inside the discovery region is more accurate than before. These are because the density $f_{J}(\cdot)$ gives more weight to the smaller size of the set $\mathbf{X}_{t}$ during the decision process. Also, it should be mentioned that the nodes outside the discovery region can be detected in a particular reception since there is no absolute and deterministic boundary for a node detection. On the other hand, under the classical approach, a total of 2 misses and 7 false alarms occur, which is 1.5 times more than the RST-based approach with $R_{0}=1 \mathrm{~km}$ and is tantamount to that with $R_{0}=0.5 \mathrm{~km}$. Note again that most of the errors under the RST-based approach, specifically the misses, come from detecting the nodes outside the discovery region which is actually a preferred error.

\section{CONCLUding REMARKS}

In this paper, we studied the problem of neighbor discovery in a wireless sensor network. By incorporating physical layer parameters, we enabled a more accurate and realistic performance assessment of the chosen neighbor discovery algorithm. Unlike the collision channel, such incorporation required us to explicitly specify the set of transmitting neighbors in each time slot based on the received signal. With the aid of the theory of random set, we were able to present an alternative method to the classical approach using a bank of matched filters for detecting the set of transmitting neighbors. The performance gain of using this new method comes in an additional cost of complexity. Several steps are still needed to complete our work. To fully validate the advantages of the alternative method, the performance evaluation needs to be supplemented with additional simulations. Also, it should be noted that we focused on discovering unidirectional links as in most of the previous work. However, for routing and other important functions of a network, bidirectional links simplify the network operation. Therefore, it is of interest to develop a self-organizing protocol which establishes bidirectional links in a distributed manner.

\section{APPENDIX A}

\section{FUndAMENTALS OF THE THEORY OF RANDOM SET}

Random set theory (RST) and its associated finite-set statistics (FISST) are extensively studied in the book Mathematics of Data Fusion [10]. This section briefly introduces the essentials of RST, and refer to [10] and other companion publications [11]-[15] for more details. In RST, the belief mass of a random finite set $\mathbf{X}$ plays a similar role to that of the cumulative distribution function of a random variable, and is defined as

$$
\beta_{\mathbf{X}}(\mathbf{C}) \triangleq \operatorname{Pr}\{\mathbf{X} \subseteq \mathbf{C}\}
$$

where $\mathbf{C}$ is a closed subset of the space $\mathbb{S}$. For example [13], if $\mathbf{X}=\{\mathbf{x}\}$, i.e., a singleton, where $\mathbf{x}$ is a random vector, $\beta_{\mathbf{X}}(\mathbf{C})=\operatorname{Pr}\{\mathbf{X} \subseteq \mathbf{C}\}=\operatorname{Pr}\{\mathbf{x} \in \mathbf{C}\}$ where $\operatorname{Pr}\{\mathbf{x} \in \mathbf{C}\}$ is the probability measure on $\mathbb{S}$. From this, it can be conjectured that the belief mass generalizes the ordinary probability measure. It is straightforward to write the belief mass in 221 as

$$
\beta_{\mathbf{X}}(\mathbf{C})=\sum_{\mathbf{B} \subseteq \mathbf{C}} \operatorname{Pr}\{\mathbf{X}=\mathbf{B}\}=\sum_{\mathbf{B} \subseteq \mathbf{C}} f_{\mathbf{X}}(\mathbf{B})
$$

where $f_{\mathbf{X}}(\cdot)$ is the belief density of a random set $\mathbf{X}$, and it plays the role of a probability density function. One natural question is how to derive $f_{\mathbf{X}}(\cdot)$ from $\beta_{\mathbf{X}}(\cdot)$ which will be answered in the sequel.

Consider the case where the hybrid space $\mathbb{S}$ is comprised only of a finite discrete space $U$. Then, the belief density $f_{\mathbf{X}}(\cdot)$ of a random finite set $\mathbf{X}$ can be obtained via the Möbius inverse transform of $\beta_{\mathbf{X}}(\cdot)$ as

$$
f_{\mathbf{X}}(\mathbf{B})=\sum_{\mathbf{C} \subseteq \mathbf{B}}(-1)^{|\mathbf{B} \backslash \mathbf{C}|} \beta_{\mathbf{X}}(\mathbf{C})
$$

by viewing sets as points in another space.

Example A.1: Take $\mathbb{S}=U=\{a, b\}$. Then, $\mathcal{P}(\mathbb{S})=$ $\{\emptyset,\{a\},\{b\},\{a, b\}\}$. Assign probability to each element of the power set so that the sum is equal to 1 :

$$
f_{\mathbf{X}}(\emptyset)=0.1, f_{\mathbf{X}}(\{a\})=0.4, f_{\mathbf{X}}(\{b\})=0.3, f_{\mathbf{X}}(\{a, b\})=0.2
$$

Then, we can obtain the values of the belief mass using (23). For example, $\beta_{\mathbf{X}}(\{b\})=f_{\mathbf{X}}(\emptyset)+f_{\mathbf{X}}(\{b\})=0.4$. Likewise, 
we have

$$
\beta_{\mathbf{X}}(\emptyset)=0.1, \beta_{\mathbf{X}}(\{a\})=0.5, \beta_{\mathbf{X}}(\{a, b\})=1
$$

We can also retrieve the values of the belief density from the belief mass using (24). For example, $f_{\mathbf{X}}(\{b\})=-\beta_{\mathbf{X}}(\emptyset)+$ $\beta_{\mathbf{X}}(\{b\})=0.3$.

For a general case where $\mathbb{S} \triangleq \mathbb{R}^{d} \times U$ with $d>0$, the Möbius inverse transform is not applicable because it applies only for a finite partially ordered set [10]. The continuous analog of the Möbius inverse transform, which is often called the set derivative, at $\mathbf{Z}=\left\{\mathbf{z}_{1}, \ldots, \mathbf{z}_{n}\right\}$ with $\mathbf{z}_{1} \neq \cdots \neq \mathbf{z}_{n}$, is defined by [14]

$$
\frac{\delta F(\mathbf{C})}{\delta \mathbf{Z}} \triangleq \frac{\delta^{n} F}{\delta \mathbf{z}_{n} \cdots \delta \mathbf{z}_{1}}(\mathbf{C}) \triangleq \frac{\delta}{\delta \mathbf{z}_{n}} \frac{\delta^{n-1} F}{\delta \mathbf{z}_{n-1} \cdots \delta \mathbf{z}_{1}}(\mathbf{C})
$$

where

$$
\frac{\delta F(\mathbf{C})}{\delta \mathbf{z}_{i}} \triangleq \lim _{\nu\left(E_{\mathbf{z}_{i}}\right) \rightarrow 0} \frac{F\left(\mathbf{C} \cup E_{\mathbf{z}_{i}}\right)-F(\mathbf{C})}{\nu\left(E_{\mathbf{z}_{i}}\right)}
$$

where $E_{\mathbf{z}_{i}}$ is a small neighborhood of $\mathbf{z}_{i}$ and $\nu(\cdot)$ is the hypervolume (i.e., Lebesgue measure) of a given set, and $\frac{\delta F(\mathbf{C})}{\delta \emptyset} \triangleq$ $F(\mathbf{C})$.

\section{APPENDIX B}

\section{DERIVATION OF (12)}

The details in deriving (12) is delivered in this section. By applying Bayes rule to the conditional probability, we have

$$
\begin{aligned}
\operatorname{Pr}\left\{k \in \mathbf{I}_{t}^{s}|| \mathbf{I}_{t}^{s} \mid=\right. & \left.h_{t}, r_{k}=r^{\prime}\right\} \\
& =\frac{\operatorname{Pr}\left\{k \in \mathbf{I}_{t}^{s},\left|\mathbf{I}_{t}^{s}\right|=h_{t}, r_{k}=r^{\prime}\right\}}{\operatorname{Pr}\left\{\left|\mathbf{I}_{t}^{s}\right|=h_{t}, r_{k}=r^{\prime}\right\}}
\end{aligned}
$$

whose numerator and denominator are specified one by one in the following. Conditioning the numerator on the number of transmitters and applying Bayes rule once again yields

$$
\begin{aligned}
& \operatorname{Pr}\left\{k \in \mathbf{I}_{t}^{s},\left|\mathbf{I}_{t}^{s}\right|=h_{t}, r_{k}=r^{\prime}\right\} \\
&=\sum_{n=h_{t}}^{J} \operatorname{Pr}\left\{\left|\mathbf{I}_{t}^{s}\right|=h_{t}\left|k \in \mathbf{I}_{t}^{s}, r_{k}=r^{\prime},\right| \mathbf{I}_{t} \mid=n\right\} \\
& \cdot \operatorname{Pr}\left\{k \in \mathbf{I}_{t}^{s}, r_{k}=r^{\prime},\left|\mathbf{I}_{t}\right|=n\right\}
\end{aligned}
$$

Define $f_{1, n}$ as the probability that a transmitter located at distance $r^{\prime}$ from the receiver will succeed among $n(\geq 1)$ simultaneous transmissions and it is given by

$f_{1, n}=1-\int_{0}^{\infty} \cdots \int_{0}^{\infty} F_{P}\left(\tau \sum_{i=2}^{n} x_{i} \mid r^{\prime}\right) d F_{P}\left(x_{2}\right) \cdots d F_{P}\left(x_{n}\right)$

Also, define $f_{2, n}$ as the probability that a transmitter at an arbitrary distance will succeed among $n(>1)$ simultaneous transmissions given that one of the other transmitters is known to be located at distance $r^{\prime}$ from the receiver which is obtained by

$$
f_{2, n}=1-\int_{0}^{\infty} \cdots \int_{0}^{\infty} F_{P}\left(\tau \sum_{i=2}^{n} x_{i}\right) d F_{P}\left(x_{2} \mid r^{\prime}\right) d F_{P}\left(x_{3}\right) \cdots d F_{P}\left(x_{n}\right)
$$

$$
\begin{aligned}
\operatorname{Pr}\left\{\left|\mathbf{I}_{t}^{s}\right|\right. & \left.=h_{t}\left|r_{k}=r^{\prime},\right| \mathbf{I}_{t} \mid=n\right\} \\
& =\frac{n}{J}\left(\begin{array}{c}
n-1 \\
h_{t}-1
\end{array}\right) f_{2, n}^{h_{t}-1}\left(1-f_{2, n}\right)^{n-h_{t}} f_{1, n} \\
+ & \frac{n}{J}\left(\begin{array}{c}
n-1 \\
h_{t}
\end{array}\right) f_{2, n}^{h_{t}}\left(1-f_{2, n}\right)^{n-h_{t}-1}\left(1-f_{1, n}\right) \\
& \quad+\left(1-\frac{n}{J}\right)\left(\begin{array}{c}
n \\
h_{t}
\end{array}\right) f_{n}^{h_{t}}\left(1-f_{n}\right)^{n-h_{t}}
\end{aligned}
$$

for all $\mathcal{S} \in \mathcal{J}_{n}^{k}$. Likewise, we can specify the remaining probabilities in (29). By noting that $\left|\mathcal{J}_{n}\right|=\left(\begin{array}{l}J \\ n\end{array}\right)$ and $\left|\mathcal{J}_{n}^{k}\right|=\left(\begin{array}{l}J-1 \\ n-1\end{array}\right)$ and after some manipulation, 29) becomes$$
\begin{aligned}
& \text { by } \\
& \mathcal{J}_{n} \\
& = \\
& = \\
& \sum_{S} \\
& \sum_{\mathcal{J}_{n}} \\
& \\
& \\
&
\end{aligned}
$$$$
\operatorname{Pr}\left\{\left|\mathbf{I}_{t}^{s}\right|=h_{t}\left|r_{k}=r^{\prime},\right| \mathbf{I}_{t} \mid=n\right\}
$$$$
=\sum_{\mathcal{S} \in \mathcal{J}_{n}^{k}} \operatorname{Pr}\left\{k \in \mathbf{I}_{t}^{s},\left|\mathbf{I}_{t}^{s}\right|=h_{t}, \mathbf{I}_{t}=\mathcal{S}\left|r_{k}=r^{\prime},\right| \mathbf{I}_{t} \mid=n\right\}
$$$$
+\sum_{\mathcal{S} \in \mathcal{J}_{n}^{k}} \operatorname{Pr}\left\{k \notin \mathbf{I}_{t}^{s},\left|\mathbf{I}_{t}^{s}\right|=h_{t}, \mathbf{I}_{t}=\mathcal{S}\left|r_{k}=r^{\prime},\right| \mathbf{I}_{t} \mid=n\right\}
$$$$
+\sum_{\mathcal{S} \in \mathcal{J}_{n} \backslash \mathcal{J}_{n}^{k}} \operatorname{Pr}\left\{\left|\mathbf{I}_{t}^{s}\right|=h_{t}, \mathbf{I}_{t}=\mathcal{S}\left|r_{k}=r^{\prime},\right| \mathbf{I}_{t} \mid=n\right\}
$$

Denote by $\mathcal{J}_{n}$ the set of elements in the power whose cardinality is equal to $n$, and node index $k$. For example, if $\mathcal{J}=\{1,2,3\}$, then

Since nodes are randomly transmitting with equal probability, each realization of the set of transmitters having same cardinalall $\mathcal{S} \in \mathcal{J}_{n}$, a over time slots (but the set of successful transmitters $\mathbf{I}_{t}^{s}$ is not). Therefore, the probability in the first summation of 29 . is computed as

$$
=\left(\begin{array}{c}
n-1 \\
h_{t}-1
\end{array}\right) f_{2, n}^{h_{t}-1}\left(1-f_{2, n}\right)^{n-h_{t}} f_{1, n} \frac{1}{\left(\begin{array}{l}
J \\
n
\end{array}\right)}
$$


where $f_{n}$ is the shorthand notation for (7). The second probability in 28) is simply

$$
\operatorname{Pr}\left\{r_{k}=r^{\prime},\left|\mathbf{I}_{t}\right|=n\right\}=\left(\begin{array}{l}
J \\
n
\end{array}\right) p_{\mathrm{T}}^{n}\left(1-p_{\mathrm{T}}\right)^{J-n} f_{r}\left(r^{\prime}\right)
$$

By substituting (31) and (32) into (28), the denominator becomes

$$
\begin{aligned}
& \operatorname{Pr}\left\{\left|\mathbf{I}_{t}^{s}\right|=h_{t}, r_{k}=r^{\prime}\right\} \\
& =\sum_{n=h_{t}}^{J}\left(\frac{n}{J}\left(\zeta_{n}+\gamma_{n}-\xi_{n}\right)+\xi_{n}\right)\left(\begin{array}{l}
J \\
n
\end{array}\right) p_{\mathrm{T}}^{n}\left(1-p_{\mathrm{T}}\right)^{J-n} f_{r}\left(r^{\prime}\right)
\end{aligned}
$$

where the shorthand notations $\zeta_{n}, \gamma_{n}$ and $\xi_{n}$ are defined in Section IV-C

\section{REFERENCES}

[1] S. A. Borbash, A. Ephremides, and M. J. McGlynn, "An asynchronous neighbor discovery algorithm for wireless sensor networks," Ad Hoc Networks, vol. 5, pp. 998-1016, 2007.

[2] S. Vasudevan, D. Towsley, D. Goeckel, and R. Khalili, "Neighbor discovery in wireless networks and the coupon collector's problem," in ACM MobiCom, Beijing, China, Sep. 2009.

[3] S. Vasudevan, J. Kurose, and D. Towsley, "On neighbor discovery in wireless networks with directional antennas," in IEEE INFOCOM, Miami, Florida, Mar. 2005.

[4] M. J. McGlynn and S. A. Borbash, "Birthday protocols for low energy deployment and flexible neighbor discovery in ad hoc wireless networks," in ACM MobiHoc, Long Beach, California, Oct. 2001.

[5] M. Zorzi and R. Rao, "Capture and retransmission control in mobile radio," IEEE Journal on Selected Areas in Communications, vol. 12, no. 8, pp. 1289 - 1298, Oct. 1994.

[6] G. D. Nguyen, A. Ephremides, and J. E. Wieselthier, "On capture in random-access systems," in IEEE ISIT, Seattle, Washington, Jul. 2006.

[7] C. Namislo, "Analysis of mobile radio slotted aloha networks," IEEE Journal on Selected Areas in Communications, vol. SAC-2, no. 4, pp. 583 - 588, Jul. 1984.

[8] B. Hajek, A. Krishna, and R. O. LaMaire, "On the capture probability for a large number of stations," IEEE Transactions on Communications, vol. 45, no. 2, pp. 254-260, 1997.

[9] S. Ghez and S. Verdú, "Stability property of slotted aloha with multipacket reception capability," IEEE Transactions on Automatic Control, vol. 33 , no. 7 , pp. $640-649$, Jul. 1988.

[10] I. R. Goodman, R. P. S. Mahler, and H. T. Nguyen, Mathematics of Data Fusion. Dordrecht, The Netherlands: Kluwer Academic Publishers, 1997.

[11] A. M. Ali, R. Hudson, F. Lorenzelli, and K. Yao, A Random Finite Set Approach to Joint Estimation/Detection/Tracking/Fusion in a Wireless Sensor Network. University of California, Los Angeles: Research Report, 2008.

[12] M. Vihola, Random Sets for Multitarget Tracking and Data Fusion. Tampere University of Technology: Licentiate Thesis, 2004.

[13] R. P. S. Mahler, "Engineering statistics for multi-object tracking," in IEEE Workshop on Multi-Object Tracking, Vancouver, Canada, Jul. 2001.

[14] _ , "Random sets: Unification and computation for information fusion-a retrospective assessment," in The 7th International Conference on Information Fusion, Stockholm, Sweden, Jul. 2004.

[15] _- Statistical Multisource-Multitarget Information Fusion. Norwood, MA: Artech House, 2007.

[16] E. Biglieri and M. Lops, "Multiuser detection in a dynamic environmentpart 1: User identification and data detection," IEEE Transactions on Information Theory, vol. 53, no. 9, pp. 3158 - 3170, Sep. 2007.

[17] D. Tse and P. Viswanath, Fundamentals of Wireless Communication. Cambridge, United Kingdom: Cambridge university press, 2005.

[18] A. Goldsmith, Wireless Communications. Cambridge, United Kingdom: Cambridge university press, 2005.

[19] A. Leon-Garcia, Probability and Random Processes for Electrical Engineering, 2nd ed. Reading, MA: Addison-Wesley Publishing Company, 1994.
[20] H. V. Poor, An Introduction to Signal Detection and Estimation, 2nd ed. New York: Springer-Verlag, 1994.

[21] XBOW MICAz Mote Specifications. [Online]. Available: http://www.xbow.com

[22] W. Stallings, Wireless Communications and Networks, 2nd ed. Upper Saddle River, NJ: Pearson Prentice Hall, 2005. 